\title{
Autismo Infantil e Estresse Familiar: Uma Revisão Sistemática da Literatura
}

\author{
Maria Angela Bravo Fávero ${ }^{12}$ \\ Manoel Antônio dos Santos \\ Universidade de São Paulo, Ribeirão Preto
}

\begin{abstract}
Resumo
Este artigo tem como objetivo avaliar sistematicamente a produção bibliográfica constituída por relatos de pesquisa indexados nas bases de dados MedL ine, Psychnfo e LIL ACS, produzida de 1991 a 2001, sobre o tema do impacto psicossocial em famillias de crianças portadoras do transtorno autista. Essa revisão propõe-se a verificar a influência deste impacto, na forma de estresse parental, como fator que afeta os cuidadores diretos e contribui para a ocorrência de alterações na dinâmica familiar. Os achados das pesquisas foram classificados de acordo com o tema investigado e os aspectos metodológicos categorizados. Os principais tópicos desenvolvidos pelos artigos foram o estresse parental nas famillias de crianças autistas, a comunicação funcional, os grupos comparativos acerca do estresse parental, as interações familiares, as estratégias de enfrentamento, a resiliência, o atendimento psicoterápico, a identificação e tratamento precoce para autismo.

Palauras-chave: Autismo; estratégias de enfrentamento; estresse; familia; revisão da literatura.
\end{abstract}

Infantile Autism and Familiar Stress: A Systematic Review of Literature

\begin{abstract}
This paper aims to evaluate systematically the literature constituted by research reports indexed in the data bases MedLine, PsycInfo and LILACS, produced from 1991 to 2001, about the psychosocial impact in families of children with autistic disorder. The review intends to access the influence of parental stress, as the factor affecting the direct caregivers and contributing to the occurrence of alterations in the familiar dynamic. The findings of the studies were classified in agreement with the investigated theme and categorized methodological aspects. The main topics focused were parental stress in the autistic children's families, functional communication, comparative groups concerning parental stress, family interactions, strategies of coping, resilience, psychotherapy, identification and early treatment for autism.

Keywords: Autism; strategies of coping; stress; family; literature review.
\end{abstract}

Os sistemas de classificação internacional das doenças, que se pretendem ateóricos, apresentam seus eixos norteadores dos critérios diagnósticos a serem utilizados nos estudos psicopatológicos. O DSM-IV (APA, 1995) situa o transtorno autista no quadro dos transtornos invasivos do desenvolvimento (TID). Segundo Classificação de Transtornos Mentais e de Comportamento (CID-10 - OMS, 1993), os indivíduos afetados por TID apresentam como características "anormalidades qualitativas nas interações sociais recíprocas e em padrões de comunicação e apresentam um repertório de interesses e atividades restrito, estereotipado e repetitivo" (p. 246). Neste grupo dos TID, de acordo com a CID-10, encontra-se o autismo infantil, autismo atípico, síndrome de Rett, outros transtornos desintegrativos da infância, transtorno de hiperatividade associado a retardo mental e movimentos estereotipados, síndrome de Asperger, outros TID e TID não-especificado.

Após a descrição de Kanner, em 1943, que denominou o quadro de autismo infantil precoce, diversos estudos sobre o autismo começaram a ser produzidos nas áreas da psiquiatria infantil, da

${ }^{1}$ Parte desse deriva-se do trabalho de conclusão de curso da primeira autora, sob orientação do segundo autor, realizado no curso de Psicologia da FFCLRP/USP, subsidiado pela FAPESP. Os autores agradecim à Prof ${ }^{a} \mathrm{Dr}^{\mathrm{a}}$ Maria Beatriz Martins Linhares, da FAMED RP/USP, pela apreciação crítica da primeira versão do manuscrito e pelas sugestões enriquecedoras.

${ }^{2}$ Endereço para correspondência: Dpt ${ }^{\circ}$. de Psicologia e Educação, NEPPSNÚCLEO de Ensino e Pesquisa em Psicologia da Saúde, FFCLRP/USP, Av Bandeirantes, 3900, 14049 130, Ribeirão Preto, SP.E-mail:masantos@ffclrp.usp.br; faveroma@hotmail.com psicologia médica, do desenvolvimento e da psicanálise. A preocupação em relação ao modo como os pais (aqui nomeados cuidadores diretos por sua presença constante ao lado do filho) percebem a criança autista sempre foi enfoque de muitos desses trabalhos. Kanner (1971; Kanner, Rodriguez \& Ashenden, 1972) fez referência, repetida pelas observações de vários outros autores, a uma possível associação do alto nível intelectual e bom nível socioeconômico encontrados em vários pais de autistas.

Inicialmente, os estudos tenderam a caracterizar os pais da criança autista como emocionalmente frios, apresentando dificuldades no estabelecimento de contato afetivo (Ornitz, Ritvo \& Gauderer, 1987). A partir de estudos mais recentes, nota-se ênfase recorrente em uma perspectiva bem diferente: os pais deixaram de ser vistos como pessoas desligadas, frias e que poderiam ter alguma característica de personalidade predisponente ao autismo de seus filhos, para serem concebidos como cuidadores que criam e se relacionam de maneira normal com suas crianças.

Essa rotação de perspectiva abriu possibilidades de investigação até então insuspeitadas ou pouco elaboradas, suscitando uma série de interrogações que se desdobram em temas e dilemas que têm merecido a atenção da literatura sistemática. Podemos nomear algumas dessas questões: haveria um padrão peculiar de resposta emocional de pais frente ao transtorno autista? Seria o estresse decorrente do convívio com a criança autista comparável ao estresse de cuidadores de crianças com outras enfermidades crônicas nas quais também se faz presente o comprometimento severo das funções psicológicas? Por meio de que mecanismos o estresse influenciaria a qualidade dos cuidados fornecidos no cotidiano familiar? Existiria um perfil típico dessas famílias, ou seja, uma associação comprovada entre 
transtorno autista e nível socioeconômico, status intelectual, educacional e ocupacional, origem étnica ou preferência religiosa?

O presente estudo propõe-se a avaliar sistematicamente a literatura científica da área através da pesquisa bibliográfica com o objetivo de investigar a ocorrência de alterações na dinâmica destas famílias, decorrente de um estresse parental crônico, buscando-se identificar temas convergentes nos artigos publicados que possibilitassem a organização e análise dos dados.

A preocupação que norteia esta revisão bibliográfica é a busca de evidências que permitam caracterizar a influência da sobrecarga decorrente dos cuidados especiais exigidos pela criança autista sobre os cuidadores diretos e o funcionamento familiar. Mais particularmente, orientamos nossa busca no sentido de verificar que perguntas são formuladas e examinadas pelo conjunto da literatura, que recursos metodológicos são privilegiados pelos estudos, sua natureza (empíricos, teóricos, relatos de experiência) e a perspectiva teórica que os informa.

\section{Método}

A pesquisa bibliográfica foi operacionalizada mediante a busca eletrônica de artigos indexados em bases de dados (MedL ine, Psyclnfo e LIL ACS - Literatura Latino-Americana e do Caribe em Ciências da Saúde), a partir de palavras-chave relacionadas ao impacto psicossocial (estresse) de famílias com crianças portadoras do transtorno autista. As consultas incluíram o período de 1991 a 2001.

A amostra compreendeu as publicações de artigos indexados em periódicos, selecionados a partir de uma leitura prévia dos resumos anexados, que seguiu os seguintes critérios de inclusão: I) veículo de publicação - optamos por periódicos indexados, uma vez que são órgãos de maior divulgação e de fácil acesso para os pesquisadores; II) idioma de publicação - artigos publicados na íntegra em língua inglesa, francesa, espanhola ou portuguesa; III) ano de publicação - foram selecionados artigos publicados entre 1991 e 2001, totalizando, portanto, um período de 11 anos; IV) modalidade de produção científica foram incluídos trabalhos originais relacionados à psicologia (relato de pesquisa, estudo teórico, relato de experiência profissional); V) referências que tiveram como objeto de estudo o casal parental com filhos em idade infantil; VI) referências que compararam o impacto psicossocial do autismo com outras patologias da infância; e, VII) referências que tiveram pertinência com o tema, tendo como critério norteador do estudo o enfoque sobre o impacto psicossocial (estresse) e a sobrecarga emocional do transtorno autista na familia, em particular nos cuidadores diretos.

Primeiramente, objetivou-se verificar a incidência de artigos publicados entre 1991 e 2001, em revistas indexadas na base de dados MedLine, sobre o tema do autismo infantil e o estresse da familia e as formas de enfrentamento. Para tanto, foram utilizados os cruzamentos dos descritores (palavras-chave) relacionados ao tema: autism and burden of care, autism and family and caregiver, autism and family and coping, autism and family and psychosocial impact, autism and family and stress, infantile autism and parent and stress. Para as revistas indexadas pela base de dados PsycInfo foram utilizados os cruzamentos das palavras-chave: autism and caregiver, autism and emotional and impact, autism and family and coping, autism and family and psychology, autism and parent and stress, autism and psychological factors. Para a verificação, no período estabelecido, das ocorrências de artigos publicados nas revistas indexadas pela base de dados LILACS, foram utilizados cruzamentos para os descritores: autismo e estratégias, autismo e estresse, autismo e familia, autismo e impacto, autismo e pais, autismo e psicologia.

Utilizando-se dos critérios para inclusão das referências, foi realizado um levantamento preliminar através da leitura seletiva dos resumos encontrados. A seguir, foram recuperados os artigos originais (selecionados) na íntegra, constituindo o corpus que delimitou o material de análise, proporcionando um tratamento mais apurado dos dados. De posse dos artigos recuperados, foi feita a leitura analítica e integral de cada estudo, a identificação das idéias-chave, a hierarquização dos principais achados e a síntese dos resultados.

Para melhor organização e compreensão, foi feito um tabulamento do material incorporado e uma análise das linhas mestras dos resultados de cada trabalho que seguiu a identificação de 14 dimensões de análise, a saber: tipo de publicação (modalidade de produção científica), ano de publicação, autores, fonte (periódicos de indexação), instituição e país de origem, temas estudados, principais objetivos/ hipóteses investigadas, descrição sumária da amostra, estratégia metodológica, tipo de análise estatística, tipo de delineamento de pesquisa, período da pesquisa e número de observações, procedimentos e instrumentos utilizados, principais hipóteses investigadas, principais resultados obtidos. Com isso, foi possível uma análise dos estudos selecionados, a fim de se obter um panorama detalhado da produção científica nacional e internacional sobre a criança autista e sua familia.

Desse modo, os artigos revisados constituíram as fontes primárias de conhecimento sobre a ocorrência de alterações na dinâmica das famílias que possuem uma criança portadora de autismo. Devido aos critérios rigorosos utilizados pelas três bases de dados consultadas, acredita-se ter englobado os artigos mais relevantes sobre o tema geral. Os trabalhos qualificados desta maneira compõem o corpus da revisão elaborada através de uma análise descritiva e qualitativa da amostra bibliográfica, a partir de uma síntese do que os autores encontraram, acompanhada de uma discussão crítica do material coligido. Em seguida, foi feito um levantamento dos temas que emergiram da leitura e análise de todos os artigos. As etapas seguidas para esse procedimento de análise temática (Triviños, 1987) envolveram: pré-análise (organização dos dados através da leitura flutuante e exaustiva de cada artigo, compreendendo a sistematização geral das principais idéias e achados sob a forma de tabelas); exploração do material (com os dados já categorizados e tabulados, procedeu-se à busca de sínteses convergentes e divergentes de idéias); interpretação dos dados (a partir das sínteses realizadas, foram selecionados os temas mais recorrentes, destacados por categorias temáticas; nessa etapa, buscou-se atribuir significados aos principais achados).

\section{Resultados}

Inicialmente serão expostos os resultados encontrados na consulta realizada com os descritores anteriormente citados, acerca da 
incidência de estudos sobre o autismo infantil e o estresse da familia, mediante consulta às bases de dados mencionadas. Os unitermos utilizados, embora indicassem algumas vezes artigos repetidos, outras vezes revelaram estudos não inclú́dos em outros descritores. Assim, foi possível garantir certa abrangência das consultas realizadas.

A base de dados MedLine possibilitou identificar um total de 65 artigos relacionados ao tema, mediante o cruzamento das palavraschave definidas previamente, dos quais 42 foram selecionados e, destes, 19 foram artigos que se repetiram. Do total de 23 selecionados (excluídos os repetidos), 22 foram recuperados.

Para a base de dados PsycInfo, os resultados dos cruzamentos resultaram em 191 referências de estudos publicados em periódicos, dos quais 36 foram selecionados e destes 12 eram repetidos. Dos 24 artigos científicos indexados, 23 foram recuperados. Vale salientar que a leitura dos resumos possibilitou verificar alguns artigos que se repetiram tanto nos diferentes cruzamentos realizados na própria base de dados, quanto da base de dados MedL ine. Mesmo assim, uma quantidade significativa de artigos foi selecionada e recuperada. Além disso, esta base de dados seleciona também livros, capítulos de livros, teses, dissertações e cartas, o que pode limitar a soma de artigos selecionados, embora também seja revelador, por outro lado, da intensa produção científica relacionada ao tema através de diversas fontes.

A base de dados LIL ACS, constituída pela literatura produzida na América Latina e Caribe, possibilitou a recuperação de um número menor de publicações. Através dos resultados da busca realizada, observa-se certa escassez de trabalhos sobre o tema no âmbito de abrangência desta base, incluindo-se as produções brasileiras. Foram 34 referências resultantes das buscas, das quais 11 foram selecionadas e, destas, sete eram artigos repetidos. Assim, dos quatro artigos selecionados, excluindo os repetidos, três foram recuperados.

A busca bibliográfica possibilitou o contato com artigos da literatura nacional e internacional na área da psicopatologia infantil, no que se refere aos trabalhos publicados sistematizados nos periódicos acerca do autismo infantil e estresse familiar. No total, foram lidos 290 resumos de artigos científicos indexados nas bases de dados citadas para seleção preliminar baseada nos títulos e conteúdo dos resumos. Foram excluídos os trabalhos que não preencheram os requisitos fixados para a revisão sistemática, sobretudo com relação aos parâmetros de pertinência e relação explícita com o tema investigado. Com a recuperação dos trabalhos selecionados, 48 artigos foram lidos na íntegra e qualificados, visando precisar sua qualidade metodológica.

As buscas indicaram maior quantidade de artigos no ano de 1992, com destaque para maior incidência de publicações nos Estados Unidos, com 32\% dos artigos. Outros países, como Bolívia, Chile, França, Holanda, Irlanda, Portugal, Singapura, Taiwan e Turquia, tiveram uma publicação cada para esta busca. Deste total de 51 artigos selecionados, $94 \%(n=48)$ dos artigos de periódicos puderam ser recuperados, constituindo o corpus desta pesquisa, tendo valor significativo enquanto estudos de natureza predominantemente teórica. Deve ser ressaltado o fato de que estes números servem apenas como uma ilustração da produção científica sobre o tema, levando-se em conta que foram descartados os artigos que se repetiram na seqüência das buscas, sendo considerados apenas aqueles incluídos a partir dos critérios estabelecidos por esta pesquisa. Este fato não descarta a possibilidade de haver uma maior incidência de estudos referentes ao autismo infantil durante os anos abrangidos pela presente revisão, porém não estruturados de acordo com o enfoque deste estudo.

Com relação aos procedimentos e instrumentos, foram encontradas pesquisas que utilizaram desde relatos clínicos, observação de sessões lúdicas de crianças na interação com os cuidadores, reuniões de aconselhamento informativo até instrumentos de avaliação padronizados, cujos temas investigados mais freqüentemente foram: funcionamento e variáveis familiares (Sprovieri \& Assumpção, 2001), estresse parental (Donenberg \& Baker, 1993; Dunn, Burbine, Bowers \& Tantleff-Dunn, 2001; Fisman \& Wolf, 1991; Kasari \& Sigman, 1997; Koegel \& cols., 1992; Konstantareas, Homatidis \& Cesaroni, 1995; Konstantareas, Homatidis \& Plowright, 1992; Rodrigue, Morgan \& Geffken, 1992); suporte social (Dunn \& cols., 2001; Henderson \& Vandenberg, 1992); enfrentamento (Dunn \& cols., 2001; Moes, Koegel, Schreibman \& Loos, 1992; Rodrigue \& cols., 1992); depressão (Donenberg \& Baker, 1993; Fisman \& Wolf, 1991; Gill \& Harris, 1991; Moes \& cols., 1992); comportamentos vistos em autistas e outros TID (Bolton \& cols., 1994; El-Ghoroury \& Romanczyk, 1999; Kasari \& Sigman, 1997; Koegel, Bimbela \& Schreibman, 1996; Konstantareas \& cols., 1992, 1995; Piven \& Palmer, 1999; Szatmari, Archer, Fisman \& Streiner, 1994). Também foi notada combinação de instrumentos padronizados, como os anteriormente referidos, com entrevista semiestruturada, observações controladas e experimentos simples dentro de uma clínica.

Na verificação sobre os recursos metodológicos dos trabalhos, com relação à modalidade de produção científica, este estudo contou com os artigos recuperados que se classificaram em: 31 relatos de pesquisa, oito estudos teóricos enove relatos de experiência profissional (sob o formato de estudos de caso). Dessa maneira, o tipo de delineamento das pesquisas empíricas que prevaleceu foram os estudos transversais, mais centrados em perguntas formuladas acerca de estresse e enfrentamento em dado momento presente, em detrimento dos estudos longitudinais. A estratégia metodológica referente ao tratamento do material coletado configurou-se, na maioria dos estudos, pelo procedimento combinado de análise quantitativa e qualitativa de dados.

Grande parte dos estudos formulou questões norteadoras relacionadas à avaliação do estresse e das estratégias de copingutilizados por.pais de crianças autistas, utilizando-se de instrumentos padronizados fundamentados em abordagens cognitivo-comportamentais. Este resultado relacionado à pesquisa dos recursos metodológicos torna-se compreensível quando examinamos os descritores essenciais utilizados neste estudo, tais como estresse e coping, enquanto construtos vinculados a aspectos de comportamento dos familiares.

Verificou-se que as pesquisas com questões norteadoras relativas à avaliação das interações familiares foram orientadas por abordagens desenvolvimentais. Contudo, as abordagens psicanalítica e sistêmica também foram constatadas nos artigos que fundamentaram uma prática de atendimento terapêutico das famílias, ao passo que a terapia comportamental foi expoente significativo enquanto estratégia de tratamento de crianças acometidas pelo autismo. 
A análise das diferentes categorias temáticas possibilitou obter um panorama geral acerca do material arregimentado e sistematizado. A seguir, o leitor poderá conferir uma análise que envolve um detalhamento dos principais achados dos artigos, o que possibilitou uma integralização de dados e a emergência de temas apresentados que propiciam informações teóricas importantes sobre o autismo e o estresse da familia, bem como o que o conhecimento científico atual traz com referência a concepções emodelos de intervenção e tratamento da criança e da familia, na promoção de melhores condições e qualidade de vida. Os temas encontrados foram: estresse parental nas familias de crianças autistas, comunicação funcional, grupos comparativos, interações familiares, estratégias de enfrentamento (coping) diante do estresse parental, resiliência, atendimento psicoterápico e compreensão do transtorno.

\section{O estresse parental nas famílias de crianças autistas}

Indubitavelmente, as familias que se encontram em circunstâncias especiais, promotoras de mudanças nas atividades de vida diária e no funcionamento psíquico de seus membros, deparam-se com uma sobrecarga de tarefas e exigências especiais que podem suscitar situações potencialmente indutoras de estresse e tensão emocional. O estresse constitui-se como uma reação psicológica, cujas fontes podem ser oriundas de eventos externos ou internos. De acordo com Lipp e Guevara (1994), o estresse pode provocar tanto sintomas físicos como psicológicos. Os possíveis efeitos psicológicos das reações ao estresse são: ansiedade, pânico, tensão, angústia, insônia, alienação, dificuldades interpessoais, dúvidas quanto a si próprio, preocupação excessiva, inabilidade de concentração em assuntos não relacionados com o estressor, inabilidade de relaxar, tédio, ira, depressão e hipersensibilidade emotiva.

Sobre este tema, nota-se um número crescente de artigos publicados a partir de 1992, o que parece demonstrar maior envolvimento dos profissionais com a questão familiar, especialmente com relação aos pais das crianças autistas. Quanto a revisões críticas de literatura, foram escassos os artigos cujo enfoque perpassa essa questão do autismo infantil e do estresse da familia (Fraser \& Murti Rao, 1991; Tunali \& Power, 1993; Estrada \& Pinsof, 1995; Travis \& Sigman, 1998; Dawson, Ashman \& Carver, 2000; Peterson \& Siegal, 2000; Kaiser, Hester e McDuffie, 2001).

Segundo Koegel e cols. (1992), as familias de autistas revelam um nível geral alto de preocupação quanto ao bem-estar de suas crianças depois que os pais não puderem providenciar mais cuidados para elas. O prejuízo cognitivo da criança é um dos focos de estresse dos pais nas suas preocupações com as inabilidades (atrasos) lingüísticas e cognitivas das crianças.

Para Tunali e Power (1993), é comum o achado de dificuldades das mães de crianças autistas em prosseguir sua carreira profissional devido ao tempo excessivo da demanda de cuidados que a criança necessita e à falta de outros cuidadores. Segundo este estudo, os autores concluem que o papel central de satisfação e desempenho está relacionado ao fato de ser mãe, enquanto a definição de bom cônjuge no caso dos pais de crianças autistas é de alguém provedor de suporte emocional e físico.

As análises de Hendersone Vandenberg (1992) das mães de crianças autistas indicaram que a gravidade do transtomo da criança (estresse), $\mathrm{o}$ suporte social da mãe (recursos), e o locus de controle percebido pela mãe (percepção) foram fatores significantes no ajustamento familiar. $\mathrm{O}$ ajuste familiar aumentou quando o evento estressor externo da gravidade do sintoma esteve menos severo e quando houve maior suporte social, que visivelmente ajudou a abrandar as dificuldades de criação da criança autista.

É importante notar que, no estudo de Moes e cols. (1992), mães de crianças autistas mostraram significativamente mais estresse do que os pais. Os autores propuseram um modelo explicativo sugerindo que o estresse pode estar relacionado às diferentes responsabilidades com a criança designadas para cada cuidador. Neste estudo, os pais estavam ativamente comprometidos com sua atividade profissional fora de casa, e todas as mães identificaram-se como o cuidador primário.

Shu, Lung e Chan (2000) investigaram o impacto de crianças autistas sobre a saúde mental de suas mães, bem como a morbidade psiquiátrica menor. Muitas familias relataram que o cuidar de uma criança autista constituiu uma sobrecarga emocional, física e financeira. Um total de 33\% de mães de crianças autistas do grupo pesquisado apresentou um transtorno psiquiátrico menor. As mães com mais anos de estudo puderam utilizar recursos melhores para procurar ajuda.

No estudo de Gray (1997), a concepção de vida familiar normal foi de difícil compreensão para muitos pais de crianças autistas de alto funcionamento e síndrome de Asperger. Seu entendimento de uma vida familiar normal estava associado a fatores tais como: suas próprias habilidades de socializar-se, a qualidade emocional de suas interações com os membros da familia, e os rituais e rotinas que abrangiam suas percepções do que fazem famílias normais. Entre as ameaças a estas atividades notou-se a presença de tendências agressivas em suas crianças.

A partir dos dados apresentados acima, foi possível detectar que o estresse dos pais de crianças autistas apareceu ligado a fatores tais como o prejuízo cognitivo da criança, a gravidade dos sintomas e as tendências agressivas do filho. Porém, um fator mediador desse estresse foi o suporte social que, quando percebido, favoreceu um melhor ajustamento familiar. Os estudos oferecem consistência à hipótese de sobrecarga emocional, física e financeira no cuidado com o portador de necessidades especiais, principalmente nas mães dessas crianças, além de uma dificuldade na representação da idéia de uma vida normal.

Vale ressaltar que a sintomatologia do estresse abrange tanto aspectos físicos como psicológicos, o que pôde ser verificado nos estudos referidos acima. Selye (1956) afirma que os indivíduos podem passar por três fases de estresse. Essas fases compreendem: a fase de alerta, em que o organismo se prepara para as reações de luta ou fuga, continuada pela fase de resistência, uma tentativa de adaptação do organismo, em que predomina a sensação de desgaste. Se o evento estressor é contínuo e o indivíduo não conta com estratégias adequadas para lidar com isto, o organismo exaure suas reservas de energia adaptativa e a fase de exaustão se manifesta (Lipp, 1989; Lipp \& Rocha, 1994).

A não ser pela menção à busca de suporte social, não foi possível apreender nos trabalhos revisados os modos ou estratégias de enfrentamento utilizados diante da situação estressora. Aparece um 
questionamento sobre a apropriação da concepção de normalidade da vida familiar. Em decorrência do acometimento da criança, acredita-se na necessidade de uma ressignificação dos papéis de cada membro da familia no processo de adaptação dos pais e da criança na situação presente.

\section{A comunicação funcional}

A comunicação é um aspecto especialmente afetado no quadro do autismo e que, com freqüência, apresenta-se severamente prejudicada. O desenvolvimento inicial da linguagem nas crianças autistas é caracterizado pela demora no início da fala ou pela falta de progresso (com possibilidade de regressão) após a aquisição inicial da linguagem. Segundo Pomeroy (1992), este atraso pode ser antecedido por uma ausência de balbucio comunicativo. Torna-se necessário que os cuidadores encontrem uma forma de tornar esta comunicação possível e funcional. De alguma maneira, a criança precisa fazer-se entender e ser entendida por aqueles que a cercam.

Trabalhos recentes têm sido desenvolvidos com o tema do autismo da criança e a familia sob o enfoque da comunicação (Barnes, Kroll, Lee, Jones \& Stein, 1998; El-Ghoroury \& Romanczyk, 1999; Fraser \& Murti Rao, 1991; Hindley, 1997; Kaiser, Hester \& McDuffie, 2001; Peterson \& Siegal, 2000; Travis \& Sigman, 1998).

El-Ghoroury e Romanczyk (1999) examinaram as interações das crianças autistas com membros da família, atentando para a manifestação da linguagem dessas crianças através de jogos. Os resultados revelaram que mães e pais de crianças autistas exibiram mais comportamentos de jogo com a criança do que os irmãos, enquanto as crianças com autismo iniciavam mais interações voltadas para os irmãos do que para os pais.

Inseridos em um contexto mais abrangente de comunicação e relacionamentos interpessoais de crianças autistas, estudos têm sido empreendidos segundo uma perspectiva cognitivo-desenvolvimental, aludindo à teoria da mente (Bosa, 2001; Fraser \& Murti Rao, 1991; Peterson \& Siegal, 2000; Travis \& Sigman, 1998). Frasier e Murti Rao (1991) definem que, nas crianças autistas, uma das anormalidades fundamentais em comunicação parece estar na inabilidade para formar representações que levem em consideração os estados mentais dos outros, o que resulta no colapso da comunicação efetiva a dois. Segundo Travis e Sigman (1998), crianças autistas têm falhas em exibir responsividade típica às emoções dos outros, o que sugere falências na função de um sistema especializado na interpretação e resposta a sinais de emoção. Muitas crianças têm dificuldade nos relacionamentos com pares, embora autistas de alto funcionamento relatem ter amigos na infância e adolescência.

Hindley (1997) centrou-se sobre a comparação de crianças com prejuízos auditivos e autistas, partindo, porém, de uma abordagem teórica diferente. $\mathrm{O}$ autor verificou, mediante revisão da literatura, que os estudos relatam que prejuízos auditivos parecem ocorrer mais freqüentemente entre crianças autistas do que seria esperado. $\mathrm{O}$ prejuízo auditivo pode confundir o diagnóstico do autismo e viceversa, podendo sucessivamente direcionar um diagnóstico tardio e manejo médico e educacional inapropriados.

Barnes e cols. (1998) verificaram que, em alguns casos, existe certa consideração de que as crianças autistas não poderiam entender os conceitos ou que poderiam ficar indevidamente angustiadas por informações acerca do que acontece na família em que está inserida, especialmente no caso de uma enfermidade que acomete o cuidador primário. Vale salientar que a falta de comunicação e esclarecimento, além da privação do cuidado, poderiam acentuaro impacto desta enfermidade do cuidador na relação com a criança.

A comunicação através de interações mostrou que crianças autistas têm dificuldades para iniciar jogos com os pais. No entanto, a utilização de jogos para auxiliar a interação entre as crianças e seus pais e irmãos, pode tornar lúdico e representar uma alternativa no enfrentamento de uma dificuldade de comunicação entre os familiares e, ao mesmo tempo, funcionar como valioso instrumento de expressão para a criança.

Atualmente, o uso de instrumentos auxiliares para a comunicação tem sido amplamente empregado em instituições brasileiras, como éo caso do Adolescent and Adult Psychoeducational Profile (APPEP) estudado por Mesibov, Schopler, Schaffer e Landrus (1988). É um modelo psicoeducacional importado dos Estados Unidos, que utiliza um programa de treinamento em comunicação (Treatment and Education of Autistic related Communication Handicapped Children-TEACCH), baseado em apoios visuais como fotografias, desenhos e palavras. Desse modo, existe uma tentativa de suprir, através das fichas, um aspecto fundamental nas vivências familiares queé a comunicação das necessidades.

\section{Os grupos comparativos e o estresse parental}

De todos os estudos empíricos recuperados e analisados, vários trabalharam com grupos comparativos entre crianças com autismo e crianças portadoras de outras patologias. A síndrome de Down apareceu como um grupo de comparação utilizado em vários estudos (Bolton \& cols., 1994; Bolton, Pickles, Murphy \& Rutter, 1998; Mickelson, Wroble e Helgeson, 1999; Piven e Palmer, 1999). De maneira geral, verificaram que tiques motores, transtomos obsessivo-compulsivos e afetivos foram significativamente mais comuns em parentes de autistas, assim como índices elevados de depressão maior e fobia social.

Mickelson e cols. (1999) examinaram as atribuições de causalidade feitas pelos pais de crianças com necessidades especiais. Os pais de crianças com síndrome de Down fizeram atribuições ao acaso genético e ao destino/vontade de Deus; pais de crianças autistas fizeram atribuições relacionadas à hereditariedade e ao ambiente; pais de crianças com retardo no desenvolvimento fizeram atribuições a problemas médicos e estresse durante a gravidez. Atribuições à própria culpa e atribuições ao ambiente (pais de autistas) foram relacionadas ao pior ajustamento, ao passo que atribuições ao destino/vontade de Deus (pais de crianças portadoras da síndrome de Down) foram associadas a um melhor ajustamento parental.

Outros estudos utilizaram como grupos comparativos familias de crianças autistas, portadores de síndrome de Down e também crianças com desenvolvimento normal (Fisman \& Wolf, 1991; Rodrigue \& cols., 1992; Sanders \& Morgan, 1997; Sprovieri \& Assumpção, 2001). Foram confirmados níveis mais elevados de estresse parental em mães e pais de autistas. Foi verificado impacto negativo sobre a saúde mental materna, sendo que o resultado depressivo afeta o desempenho como mãe e cônjuge.

Donenberg e Baker (1993) compararam o impacto nas familias de crianças pré-escolares com problemas de comportamento (agressividade, 
hiperatividade), sem problemas significantes de comportamento e autistas, e verificaram que os pais de crianças com problemas de comportamento relataram níveis de impacto e estresse tão altos quanto aqueles referidos por pais de crianças com autismo. Em relação às medidas mais extensas de bem-estar parental e conjugal, entretanto, os três grupos de familias de pré-escolares não diferiram.

Pode-se concluir, a partir do estudo de Sprovieri e Assumpção (2001) que, considerando famílias de autistas, de portadores de síndrome de Down e de filhos saudáveis quanto à dinâmica familiar, as famílias dos autistas e portadores de síndrome de Down são dificultadoras da saúde emocional dos elementos do grupo, constituindo as dos autistas maior porcentagem, com presença de estresse nesses pais.

Foi possível verificar, ainda, outros autores que utilizaram grupos comparativos, não das crianças, mas para estimar a extensão de concordância entre pais e professores sobre sintomas autistas e habilidades comportamentais adaptativas de crianças autistas (Szatmari \& cols., 1994).

De maneira geral, as crianças portadoras da síndrome de Down constituíram um grupo de comparação extensamente utilizado em trabalhos sobre autismo. Através dos estudos revisados verificou-se que tiques motores e transtornos obsessivo-compulsivos (TOC) e afetivos foram significativamente mais comuns em parentes de autistas do que de síndrome de Down. Foram verificados índices aumentados de transtornos afetivos (especialmente transtorno depressivo maior) e fobia social em parentes de primeiro grau de autistas, embora não exista evidência que indique significante comorbidade entre transtorno afetivo e o fenótipo do autismo.

\section{Interações familiares e estresse parental nos grupos comparativos}

Alguns trabalhos utilizaram-se de sessões de interação entre o cuidador e a criança afetada para avaliar o estresse dos pais de crianças autistas, comparando-o com outras patologias (El-Ghoroury \& Romanczyk, 1999; Kasari \& Sigman, 1997; Nally, Houlton \& Ralph, 2000; Willemsen-Swinkels, Buitelaar \& van Engeland, 1997). Pais que relataram maior estresse tinham crianças autistas que eram menos responsivas nas interações sociais. Foi essencial verificar que, quando os pais estruturaram o comportamento de suas crianças, isto resultou em um aumento de ofertas sociais e olhares por parte delas, podendo ser observada a estruturação como um estilo de enfrentamento que se correlaciona com a maior adaptação dessas crianças.

El-Ghoroury e Romanczyk (1999) sugerem que os pais poderiam compensar o nível de inabilidade de suas crianças pela iniciação em mais jogos de interação, o que implica ensinar os pais como recorrer a tais interações para que, posteriormente, as crianças possam iniciálas por iniciativa própria.

Atualmente vários trabalhos, inclusive no contexto brasileiro, embora não enfocando especificamente a questão do autismo, têm sido produzidos a partir de diferentes perspectivas acerca da análise da interação pais-bebê/criança (Rossetti Ferreira, 1984; Piccinini \& cols., 2001).

As interações familiares constituíram importantes fatores a serem analisados, uma vez que aproximaram o pesquisador da realidade do ambiente familiar oferecido à criança e da forma com que as interações ocorrem. Foi verificado que, quando os pais estruturaram o comportamento da criança, esta apresentou maior adaptação, salientando-se a importância de os pais se engajarem em jogos com suas crianças. Este achado possibilitou intervenções visando à estimulação da criança.

\section{As estratégias de enfrentamento (coping) diante do estresse parental}

Entende-se por coping as habilidades desenvolvidas para o domínio e adaptação às situações de estresse. $\mathrm{O}$ coping tem duas funções: centrado no problema, quando modifica as relações entre pessoas e ambiente, e o centrado na emoção, cujo intuito é adequar a resposta emocional ao problema (Savóia, 1999).

A construção de estratégias de enfrentamento constitui-se como ponto fundamental a ser investigado no presente estudo. Sustenta esta investigação o fato do(a) filho(a) possuir uma condição especial de comportamento e padrão de interação não só com a mãe, mas também com os demais membros da familia, considerando também o âmbito social de relacionamentos interpessoais. Foi possível verificar algumas estratégias tanto no sentido de abrandamento de sintomas dos filhos, quanto na tentativa de redução da sobrecarga emocional dos pais.

\section{Aconselhamento informativo}

Kuloglu-Aksaz (1994) buscou avaliar se o aconselhamento informativo acarretaria em menor estresse por parte dos pais de crianças com autismo. Como resultado, os pais do grupo experimental mostravam-se mais realistas sobre as possibilidades e limites de suas crianças autistas e aceitavam mais a solidariedade e o suporte de união com outros pais, bem como manifestaram um sentido crescente de otimismo nas interações estabelecidas em casa e na comunidade. Os pesquisadores acreditam que o processo de aconselhamento informativo positivo e de desenvolvimento com outros pais deveria começar no mesmo momento em que o diagnóstico é estabelecido.

\section{Programas de treinamento de pais}

Powers (1991) propõe que, no treinamento comportamental dos pais, três áreas adicionais devem ser consideradas na avaliação: a suficiência técnica das habilidades de modificação de comportamento dos pais, os recursos da familia e sua prontidão para mudança, e o estilo instruído dos pais, com ênfase especial nos padrões entre gerações. Como tendências futuras, o autor aponta que a integração da terapia comportamental e os modelos de sistema familiar para o propósito de avaliação e tratamento de familias de crianças jovens com severa incapacidade representa uma mudança na conceitualização de intervenções com essa população.

Contudo, Koegel e cols. (1996) avaliaram se estilos parentais globais de interações durante atividades domésticas não estruturadas poderiam ser diferencialmente afetados por dois tipos muito diferentes de treinamento parental. $\mathrm{O}$ paradigma focado sobre o ensinamento individual de comportamentos-alvo não produziu influência significante nas interações do pré e pós-treinamento. Em contraste, o paradigma de treinamento parental focado no 
ensinamento de respostas centrais de motivação e responsividade para múltiplas situações resultou na demonstração de interações pais-filhos avaliadas como mais felizes, em que os pais se interessaram mais pela interação, que foi menos estressante, e o estilo de comunicação foi mais positivo. Os resultados sugerem que o tipo de treinamento parental pode ter influência positiva na redução do estresse da vida diária.

Outra estratégia presente na literatura foi a utilização de apoios visuais auxiliando no tratamento de crianças autistas. De acordo com Dettmer, Simpson, Myles e Ganz (2000), educadores e cuidadores podem usar suportes visuais com razoável confiança, pois são instrumentos fáceis de implementar em vários ambientes, além de baratos e úteis, que podem trazer resultados positivos para essas crianças.

Com base em pesquisa sobre o efeito do autismo nas famílias e nos profissionais, Jones (1997) elaborou uma lista de recomendações gerais para aprimorar a prática dentro de escolas e unidades de serviços para crianças com autismo e síndrome de Asperger. Dentre eles, estão: buscar informação sobre autismo e síndrome de Asperger e suas implicações educacionais e discutir abertamente com pais o conhecimento que eles têm de sua criança e as informações sobre autismo, bem como trocar informação sobre os serviços e instalações para crianças com autismo e suas famílias.

\section{Medicações}

No caso do autismo, segundo Klein e Slomkowski (1993), algumas medicações - tais como neurolépticos (haloperidol, clorpromazina, tioridazina) - oferecem marcada melhora na agitação psicomotora e nos comportamentos descontrolados de crianças autistas. Estas medicações não revertem a falta de responsividade (sensibilidade) social e comunicação, características-chave do autismo, mas podem facilitar a permanência dessas crianças em casa sob os cuidados da família. A perspectiva de melhorar a qualidade de vida destas crianças pode ser alcançada em muitas instâncias com o tratamento conjunto dos pais e da criança.

Sobre as medicações usadas pelas crianças portadoras do transtorno autista, a presença ou ausência de linguagem, irritabilidade, idade mental, tamanho da família e estresse familiar foram consideradas por Konstantareas e cols. (1995) como variáveis que poderiam estar relacionadas ao fato de os pais darem ou não medicamentos aos filhos portadores do transtorno autista. Fatores como o estresse familiar, a capacidade de fala da criança e a irritabilidade foram bons potenciais discriminadores para diferenciar a opção dos pais pela medicação de seus filhos, mas não a gravidade da sintomatologia. As variáveis que demonstraram maior poder preditivo para a opção dos pais pela medicação relacionam-se à capacidade para manejar a criança com transtorno autista. No exame do estresse parental como uma função da variedade de características da criança, encontramos que a irritabilidade pôde predizer estresse parental com maior probabilidade do que outras características, como a gravidade dos sintomas de transtorno autista.

\section{Suporte social como alternativa}

O suporte social tem mostrado terimportante influência na dinâmica familiar da criança autista. Konstantareas (1991), através de uma revisão da literatura sobre eventos estressores, verificou que foram relatados nos estudos como muito estressantes para os pais não apenas características físicas da criança, mas estranhezas comportamentais na forma de elas se estimularem e no uso inapropriado do corpo, batendo as mãos, cheirando e colocando a boca em objetos, balançando-se, girando e olhando fixamente os dedos. A gravidade da sintomatologia parece estar positivamente correlacionada com o estresse. Como estratégias de enfrentamento, Konstantareas ressalta o acesso à assistência profissional competente, bons programas educacionais, estilo de vida familiar confortável e acesso à disponibilidade de uma ajudante (babá), que deveriam tornar mais manejável a experiência estressante da não funcionalidade da criança, oferecendo apoio tanto no plano material como psicológico.

Davies (1996) pesquisou sobre o papel de uma ajudante de familia (family services worker) como alguém que poderia atenuar o estresse dos pais. Os resultados indicaram que muitas familias com uma criança de espectro autista são colocadas em considerável tensão por causa da falta de facilidades e serviços de apoio disponíveis para ajudar no alívio de pressão. Para Davies, enfermeiros são bem qualificados para desempenhar o papel de ajudante na família de indivíduos com incapacidade de aprendizagem ou mental.

Para Dunn e cols. (2001), estilos de enfrentamento diversos em mães e pais de crianças com autismo corresponderam a desfechos negativos (depressão, isolamento social e problemas de relacionamento conjugal). Altos níveis de apoio social corresponderam a menores índices de problemas conjugais. Eventos estressores não foram preditores diretos de resultados negativos quando sua influência foi moderada pelo suporte social e estilo de enfrentamento.

Perry e Black (1997) concluíram, mediante investigação sobre a colocação de familias com crianças autistas em atividades externas à casa, que serviços tradicionais de apoio familiar, tais como aconselhamento, terapia de casal e treinamento dos pais, foram relatados como atitudes importantes para auxiliar a familia no melhor enfrentamento da condição da criança em casa. Os autores enfatizam a importância da parceria entre pais e programas de apoio no planejamento das metas para o desenvolvimento da criança, como perspectiva potencial futura, afetando os pais quanto à colocação nas atividades externas à casa.

Coulthard e Fitzgerald (1999) investigaram, através de um questionário, a religião organizada e as convicções pessoais enquanto recursos e estratégias de enfrentamento, e sua relação com a saúde geral. Os resultados mostraram que pais de crianças com transtorno autista receberam significativamenete menos apoio de suas religiões organizadas do que das crenças pessoais, particularmente o uso de orações, que foi associado com melhor saúde geral.

Um fator importante verificado concerne ao desenvolvimento de estratégias de enfrentamento (coping), isto é, as habilidades que serviram ao domínio e adaptação às situações de estresse, suscitadas nas familias mediante a criação de alternativas de satisfação. Diante do estresse parental vivenciado nas famílias de crianças autistas, a utilização de 
aconselhamento informativo enquanto estratégia de enfrentamento foi considerada positiva, com orientação a ser fornecida desde o momento do diagnóstico. Os programas de treinamento de pais puderam auxiliar no manejo das crianças. Porém, diferentes treinamentos parentais resultaram em afeto expressivo diferente associado aos modelos de interação paiscriança, ainda que o afeto não fosse alvo de nenhum dos treinamentos parentais e que o tipo de treinamento parental possa ter influência positiva na redução do estresse da vida diária. A aderência ao uso de medicamentos, tais como neurolépticos, foi citada e seu emprego esteve mais relacionado à irritabilidade da criança do que propriamente à gravidade da sintomatologia. Contudo, o fator da severidade do sintoma esteve positivamente correlacionado com o estresse nos pais, que por sua vez pôde ser atenuado com o suporte social, especialmente com a presença de um ajudante para os cuidados com a criança.

\section{Resiliência}

As pessoas reagem de modo particular diante de situações de vida estressoras. O coping é uma dessas formas de reação peculiar que as pessoas desenvolvem para lidar com crises e adversidades, no contexto de sua cultura, sociedade e época, aliviando os aspectos negativos das situações de estresse. A resiliência pode ser definida como a resistência ao estresse, apesar da exposição a eventos estressores.

Estresse e copingsão conceitos que aparecem interligados nas pesquisas sobre resiliência. A contrapartida do estresse éo conceito de coping. Yunes e Szymanski (2001, p. 31) resumem esse dualismo da seguinte maneira: estresse é o pólo negativo e coping, o pólo positivo da resiliência. Os estudos sobre coping mostram que os cuidadores diretos (pais ou seus substitutos) acabam por criar formas para enfrentar as dificuldades de comunicação com as crianças autistas, possibilitando-lhes alternativas de satisfação. Tunali e Power (1993) observaram este processo em famillias de crianças portadoras de deficiência. De forma análoga, Moes e cols. (1992) investigaram perfis de estresse no casal parental de cuidadores de crianças autistas, encontrando maior incidência de estresse nas mães.

Pereira (2001) afirma que os mecanismos de coping estão intimamente relacionados com resiliência (em inglês, aparece eventualmente o termo hardiness, que literalmente significa audácia, intrepideze também forra, vigor). Desse modo, habilidades de coping e personalidade resiliente proporcionam maneiras de lidar com tais situações eliciadoras de estresse. A autora propõe que o indivíduo resiliente tem perspectivas que o tomam capaz de ultrapassare cicatrizar de uma forma natural, dinâmicae construtiva as dificuldades da vida (Pereira, 2001, p. 78).

Segundo Ganellen e Blaney (1984) indivíduos com personalidade ousada, valente, resistente ( $h a r d y)$, podem permanecer mais saudáveis depois de experiências intensas de estresse por possuírem características que os diferenciam de indivíduos mais vulneráveis a efeitos negativos do estresse è enfermidades físicas. Gill e Harris (1991) propõem que a personalidade resiliente (hardiness) de mães de crianças autistas contribui como atenuante do estresse vivenciado por elas.

Através do estudo de Gill e Harris (1991), verificou-se que mães de autistas que perceberam o apoio social como mais disponível experimentaram significativamente menos estresse, comprometimentos somáticos e sintomas depressivos do que aquelas com menos percepção deste apoio. A resiliência foi um bom preditor de ambos os sintomas (depressivos e comprometimentos somáticos). O referido estudo fornece apoio a achados prévios sobre a importância da personalidade resiliente como fator atenuante contra o estresse. As dimensões de resiliência foram processos importantes na adaptação a eventos estressores. Indivíduos que se apóiam em valores que orientam suas vidas tendem a enfrentar melhor a presença de um membro incapacitado na família do que aqueles que não têm crenças.

Uma das implicações da associação observada entre resiliência e suporte social percebido é mostrar que essas duas variáveis correlacionam-se. Assim, para Gill e Harris (1991), parece plausível que indivíduos que percebem altas intensidades de suporte social sintam-se mais resistentes e fortalecidos. A resiliência pode, assim, ser considerada fator de empoderamento em mães de crianças autistas. Considerando que este é um conceito que se refere a resultados positivos diante de uma situação adversa, como o fato de se ter em um filho autista, as mães resilientes foram aquelas que receberam apoio social em determinado momento, o que as tornou mais adaptadas a essa situação.

Atendimento psicoterápico e compreensão do transtorno

$\mathrm{O}$ atendimento psicológico de crianças com TID pode ser caracterizado por dificuldades peculiares com relação à compreensão da linguagem utilizada por elas, que muitas vezes não passa pela instância verbal. Segundo Lino Silva (1998), o processo de atendimento psicológico dessas crianças pode ser iniciado com tentativas de se encontrar uma linguagem comum com a criança, uma forma de comunicação de modo a que se possam partilhar experiências (p. 61).

Dentre as buscas bibliográficas do presente estudo, foram escassos os trabalhos publicados que focalizaram aspectos dos atendimentos individuais de crianças autistas, embora exista uma produção significativa na área. Cabral (1994) elaborou um estudo teórico enfatizando a compreensão, através da abordagem psicanalítica, das perturbações da personalidade precocemente instaladas, tais como o autismo e a psicose.

Estudos recentes apresentam modelos de intervenção (Catafesta, 1992) provocadores de mudanças significativas no estresse vivenciado pelos cuidadores quando inseridos em atendimentos psicológicos (Morales, Martinez \& Váldes, 1995; Prado, 1999). Kupfer (1997) relata que o trabalho institucional de atendimento grupal a pais tem se revelado muito significativo e para muitos pais, o que se verifica é um abandono da posição de culpa a a adoção de uma posição na qual se responsabilizam por seus filhos (p. 88).

Através da revisão da literatura, Estrada e Pinsof (1995) verificaram que as famílias de crianças portadoras de transtornos comportamentais da infância que respondem melhor ao tratamento têm crianças menos prejudicadas cognitivamentedurante o princípio do tratamento. São famílias que podem se engajar ativamente em muitos programas exigentes e que acompanham a escola, que pode apoiar o empenho do treinamento dos pais em casa. 
Segundo Amy (2001), o anseio dos pais é encontrar um espaço que possa fornecer acolhimento terapêutico e educativo que, ao ajudar seus filhos, permita-lhes continuar sua vida, trabalhar e dar aos outros filhos, quando eles existem, o tempo e o amor necessários (p. 91). O contato com outros pais se mostra necessário para uma troca de experiências e vivências com a finalidade de evitar a repetição de erros, de dificuldades ou problemas (Gauderer, 1987).

Prado (1999) verificou os meios de comunicação utilizados em familias com crianças autistas através da psicoterapia com a criança em paralelo à terapia familiar psicanalítica. Foram percebidas modificações quando a comunicação afetiva se abriu para a mãe, permitindo contato mais verdadeiro com sua realidade psíquica, o que refletiu na retomada do desenvolvimento do filho. Deste modo, a terapia para ambos pode ser entendida com uma estratégia de adaptação e enfrentamento da mãe e criança em uma situação que inclui um fator estressante.

Sidoli (2000) descreveu um processo psicoterápico em que a terapeuta auxiliou a mãe a entender as comunicações e necessidades da criança, de modo que pudessem fazer sentido para ela, além de aceitar as diferenças da criança e de si própria. A criança pôde iniciar o desenvolvimento da linguagem como um caminho de alcance de sua mãe em uma via simbólica, e para começar a sair da concha de defesas autistas na qual ela tinha sido encapsulada.

Reid (1999) descreveu o trabalho feito com as familias e as crianças autistas na Tavistock Clinic, em Londres, a partir de uma abordagem psicodinâmica. $O$ foco esteve na descoberta da localização do estresse na família, na natureza daquele estresse e na exploração das intervenções terapêuticas possivelmente prestativas. Reid propôs um modelo de avaliação da criança e da família que inclui algumas fases, como a observação participante e o aprendizado da história da criança, a avaliação da criança e das necessidades dos pais e dos irmãos, e um plano de tratamento para qualquer familiar que necessite de suporte psicológico, com avaliação contínua.

Morales e cols. (1995) apresentaram um caso clínico de atendimento de uma criança autista e sua família, analisado sob o enfoque interacional da teoria sistêmica. Concluíram que este enfoque tem contribuído para compreender a responsabilidade do grupo familiar, tanto na aparição e manutenção do sintoma, como na cura e manutenção da melhora. A estratégia de enfrentamento, como no estudo anterior, parece estar centrada nos atendimentos, sendo, neste caso, com um enfoque sistêmico.

As abordagens psicodinâmicas e o enfoque sistêmico prevaleceram enquanto suportes teórico-técnicos de intervenção com crianças autistas e suas famílias, especialmente as mães. Os resultados explicitaram modificações passíveis de serem obtidas quando mãe e filho foram acompanhados por atendimento psicológico individual ou familiar.

Preconiza-se que a relação terapêutica possa implicar em uma reconstrução das relações significativas com os outros através do contato com o terapeuta. De um ponto de vista psicodinâmico, através de sua escuta atenta o terapeuta pode entrar em contato com a história desses pais, com seus ideais, suas frustrações e idealizações. Então, abre-se um espaço para lidar com as crenças relacionadas à condição especial do filho.

\section{Autismo: identificação e tratamento precoce}

Nessa categoria foram inseridos os trabalhos que, embora não enfocassem diretamente a questão do estresse familiar, trouxeram informações consideradas importantes para o estudo mais amplo dessa patologia da infância. Ornitz e cols. (1987) afirmam que, atualmente, em linhas gerais, os trabalhos existentes não mostram nenbuma diferença significativa entrepais de autistase outros (p. 125), acrescentando que o transtorno autista pode ocorrer em familias de qualquer nível sócio-econômico, intelectual, ocupacional, educacional, racial, étnico ou religioso.

Outros temas foram: dor crônica em pais de crianças com inabilidades (Burke, Hainswoth, Eakes \& Lindgren, 1992), experiências precoces desadaptativas (Dawson \& cols., 2000), investigação de sintomas do transtorno autista (Kracke, 1994; Whiteley, Rodgers \& Shattock, 1998), entrevista diagnóstica (Lord, Rutter \& Le Couteur, 1994), etiologia (Bernard-Opitz, Kwood \& Sapuan, 2001), construção imaginária que os pais fazem a respeito da criança com transtorno de espectro autista (Avdi, Griffin \& Brough, 2000).

Discutindo sobre o desenvolvimento da habilidade de atenção compartilhada e suas implicações para a identificação precoce do autismo, Bosa (2002) apontou a existência de controvérsias nos estudos sobre o desenvolvimento inicial de bebês posteriormente diagnosticados como autistas. Segundo a autora, as pesquisas não possibilitaram evidenciar comprometimento nos primeiros meses, seja utilizando a observação de vídeos domésticos ou informações fornecidas pelos pais.

Hall (1992) verificou a literatura sobre o tratamento precoce de transtornos incapacitantes permanentes, tais como paralisia cerebral, retardo mental e autismo. Foi reconhecido que estas condições não são curadas por tratamentos precoces, mas a qualidade de vida pode ser melhorada na medida em que a adaptação, habilidades de enfrentamento e funcionamento familiar são facilitados.

Howlin e Clements (1995) também desenvolveram um estudo muito particular, deparando-se com uma questão central: é possível avaliar o impacto de maus tratos em crianças com TID? O objetivo era verificar, através dos comportamentos infantis tais como relatados pelos pais, as denúncias de abuso em uma escola especializada em autismo. Fobias gerais mostraram algum aumento, mas a mudança mais notável foi o medo e a resistência em ir para a escola. Comportamentos auto-agressivos e prejudiciais, transtorno de humor, níveis de atividade e acessos de raiva aumentaram e, embora não houvesse perda particular de interesse em atividades diárias, distúrbios do sono foram comuns. Várias crianças mostraram um tipo específico de problema com alimentação, não usual em autistas e que não haviam mostrado previamente, que consistia em agarrar e arremessar comidas e bebida. A consistência nos tipos de dificuldades de comportamento relatadas e no tempo em que houve aumento dos transtornos comportamentais sugere que a criança sofreu marcado estresse emocional seguido às suas experiências na escola. Como o modelo diferencial de mudança foi tão similar nas crianças, parece improvável que a narrativa dos pais tenha sido simplesmente fabricada.

Com relação à identificação e tratamento precoce do autismo, mesmo através de vídeo e informação dos pais não foi possível 
verificar o comprometimento de tipo autista nos anos iniciais da criança. Embora o tratamento precoce não tenha curado transtornos incapacitantes da criança, como o autismo, a qualidade de vida pôde ser melhorada através dessa estratégia.

\section{Considerações Finais}

Nainterlocução comolegado da literatura, percebe-se que são diversas as inquietações e que alguns desafios, dilemas e dúvidas que compartilhamos no passado ainda permanecem no estágio atual do conhecimento científico.

A associação entre um perfil típico das familias de crianças autistas e um nível socioeconômico, statusintelectual, educacional e ocupacional, origem étnica ou religiosa não foi confirmada. Contudo, os estudos revisados ressaltam que, a despeito da falta de um padrão peculiar de resposta, a presença recorrente do achado da condição de ter como um de seus membros uma criança portadora de transtorno autista constituiu fonte eliciadora de estresse nos pais, que acarreta uma sobrecarga, principalmente, de natureza emocional. Embora a severidade do sintoma seja fonte de estresse na familia, o prejuízo cognitivo foi o principal fator de estresse parental. Neste sentido, torna-se possível refletir que uma indeterminação quanto ao futuro, especialmente com relação aos comportamentos de independência, desde atividades de vida diária e prática da criança até vida social e escolar, suscitam nos pais questionamentos com relação aos cuidados de seus filhos no futuro, quando eles próprios não mais puderem provê-los.

O estresse, o enfrentamento e a resiliência foram construtos teóricos que apareceram interligados nessa revisão da literatura. Diante de uma condição peculiar de sobrevivência, como é o caso da familia que convive com uma criança autista, a dinâmica familiar sofre mobilizações que vão desde aspectos financeiros até aqueles relacionados à qualidade de vida física, psíquica e social dos cuidadores diretos. Os pais têm que fazer o luto pela perda do filho ideal, para que possam perceber as reais capacidades e potencialidades de sua criança. O emprego do termo resiliência que prevaleceu foi enquanto uma boa adaptação em condições desfavoráveis, adversas, em que o indivíduo apresentou resultados adaptativos.

Concomitantemente, a literatura aponta o modo como esses pais se defrontam com as atividades estereotipadas da criança, o isolamento, a ausência do brincar, que acaba culminando, muitas vezes, com o próprio afastamento familiarde uma vida social. As preocupações com agravidade dos sintomas e com a agressividade do filho fazem do estresse da familia com criança autista ser maior quando comparado com famílias com suas crianças portadoras de outras enfermidades, tais como a síndrome de Down. A dificuldade diagnóstica, a multicausalidade desse transtorno e a ausência de um componente genético definido no caso do autismo podem estar relacionados a um sentimento de culpa verificado em pais de autistas.

Do reconhecimento destes sentimentos, torna-se necessária uma tomada de responsabilidade ativa dos pais no sentido da elaboração de estratégias de enfrentamento da situação. Neste sentido, os aconselhamentos, orientações, apoio social através das instituições de atendimento às crianças, principalmente, e os trabalhos terapêuticos emergem como alternativas de enfrentamento.
A despeito deste impacto, cuidadores que vivem situações semelhantes nem sempre são afetados da mesma maneira, ainda que todos estejam diante dessa sobrecarga. O conceito de resiliência foi inserido nesse contexto. Porém, é um novo construto na área psicológica e sua utilização ainda é restrita ao ambiente acadêmico. Os artigos que trabalharam com o construto de resiliência, utilizaram-no enquanto a boa adaptação dos indivíduos diante de condições adversas, ou seja, obtenção de resultados positivos e de sucesso em condições desfavoráveis que ofereciam risco à saúde mental.

Esta abordagem do material recuperado constituiu-se apenas em um recorte da literatura científica sobre estresse e autismo. Ressalta-se a importância de novos estudos que tragam questionamentos sobre as alternativas oferecidas e/ou buscadas destes cuidadores para o enfrentamento da situação de adoecimento crônico do filho, em âmbito nacional. E, neste sentido, conhecer melhor as necessidades tanto psicológicas quanto relacionadas a políticas públicas que visem atenuaro sofrimento emergente nessa condição. Aconselhamentos sobre planejamento de_mudanças nos cuidados das crianças, assim como o engajamento em atividades recreacionais, ampliando a rede de suporte familiar,poderia reduzir a probabilidade de dificuldades comportamentais, atenuando a ansiedade e fortalecendo a apropriação de estratégias de enfrentamento positivas.

O conhecimento gerado pela presente revisão constitui um recorte que contribui para uma maior compreensão da situação atual das familias que têm, como um de seus membros, uma criança portadora do transtorno autista e, de maneira mais ampla, portadores de transtornos invasivos do desenvolvimento. Ainda que não tenha sido objetivo do presente estudo esgotar o assunto, fica a convicção de que a revisão efetuada oferece suporte teórico e empírico para o argumento de que trabalhos de intervenção em diferentes abordagens, baseados em uma reflexão desenvolvida a partir de diferentes referenciais de leitura e enfoques teóricos, podem abrir novos rumos para os desafios colocados pelo autismo na contemporaneidade.

\section{Referências}

Amy, M. D. (2001). Enfrentando o autismo: A criança autista, seus pais e a relação terapêutica. Rio de Janeiro: Zahar.

Associação Americana de Psiquiatria (1995). DSM-IV, Manual diagnóstico e estatístico de transtornos mentais ( $4^{a}$ ed. revisada; D. Batista, Trad.). Porto Alegre: ArtMed.

Avdi, E., Griffin, C. \& Brough, S. (2000). Parents'constructions of the 'problem'during assessment and diagnosis of their child for an autistic spectrum disorder. Journal of Health Psychology, 5, 241-254.

Barnes, J., Kroll, L., Lee, J., Jones, A. \& Stein, A. (1998). Communication about parental illness with children who have learning disabilities and behavioural problems: Three case studies. Child Care Health Developmental, 24, 441-456.

Bernard-Opitz, V., Kwood, K. W. \& Sapuan, S. (2001). Epidemiology of autism in Singapore: Findings of the first autism survey. International Journal of Rehabilitation Research, 24, 1-6.

Bolton, P., Macdonald, H., Pickles, A., Rios, P., Goode, S., Crowson, M., Bailey, A. \& Rutter, M. (1994). A case-control family history study of autism. Journal of Child Psychology and Psychiatry and Allied Disciplines, 35, 877-900.

Bolton, P. F., Pickles. A., Murphy, M. \& Rutter, M. (1998). Autism, affective and other psychiatric disorders: Patterns of familial aggregation. Psychological Medicine, 28, 385-395. 
Bosa, C. A. (2001). As relações entre autismo, comportamento social e função executiva. Psicologia: Reflexão e Crítica, 14, 281-287.

Bosa, C. (2002). Atenção compartilhada e identificação precoce do autismo. Psicologia: Reflexão e Crítica, 15, 77-88.

Burke, M. L., Hainsworth, M. A., Eakes, G. G. \& Lindgren, C. L. (1992). Current knowledge and research on chronic sorrow: A foundation for inquiry. Death Studies, 16, 231-245.

Cabral, M. F.S. (1994). Contribuições da psicanálise para a psicologia e teoria do conhecimento. Revista Portuguesa de Psicanálise, 13, 45-57.

Catafesta, I. F. M. (1992). Intervenções no desenvolvimento psicológico: Um trabalho preventivo. Tese de Doutorado não-publicada, Instituto de Psicologia, Universidade de São Paulo. São Paulo, SP.

Coulthard, P. \& Fitzgerald, M. (1999). In God we trust? Organised religion and personal beliefs as resources and coping strategies, and their implications for health in parents with a child on the autistic spectrum. Mental Health, Religion \& Culture, 2, 19-33.

Davies, J. (1996). The role of the specialist for families with autistic children. Nursing Standard, 11, 36-40.

Dawson, G., Ashman, S. B. \& Carver, L. J. (2000). The role of early experience in shaping behavioral and brain development and its implications for social policy. Development and Psychopathology, 12, 695-712.

Dettmer, S., Simpson, R. L., Myles, B. S. \& Ganz, J. B. (2000). The use of visual supports to facilitate transitions of students with autism. Focus on Autism and other Developmental Disabilities, 15, 163-169.

Donenberg, G. \& Baker, B. L. (1993). The impact of young children with externalizing behaviors on their families. Journal of Abnormal Child Psychology, 21, 179-198.

Dunn, M. E., Burbine, T., Bowers, C. A. \& Tantleff-Dunn, S. (2001). Moderators of stress in parents of children with autism. Community Mental Health Journal, 37, 39-52.

El-Ghoroury, N. H. \& Romanczyk, R. G. (1999). Play interactions of family members towards children with autism. Journal of Autism and Developmental Disorders, 29, 249-258.

Estrada, A. U. \& Pinsof, W. M. (1995). The effectiveness of family therapies for selected behavioral disorders of childhood. Journal of Marital and Family Therapy, 21, 403-440.

Fisman, S. \& Wolf, L. (1991). The handicapped child: Psychological effects of parental, marital, and sibling relationships. Psychological Clinics of North America, 14, 199-217.

Fraser, W. I. \& Murti Rao, J. (1991). Recent studies of mentally handicapped young people's behaviour. Journal of Child Psychology and Psychiatric and Allied Disciplines, 32, 79-108.

Gauderer, E. C. (1987). Crianças, adolescentes e nós: questionamentos e emoções. São Paulo: Almed.

Gill, M. J. \& Harris, S. L. (1991). Hardiness and social support as predictors of psychological discomfort in mothers of children with autism. Journal of Autism and Developmental Disorders, 21, 407-416.

Gray, D. E. (1997). High functioning autistic children and the construction of "normal family life". Social Science and Medicine, 44, 1097-1106.

Hall, D. M. (1992). Child health promotion, screening and surveillance. Journal of Child Psychology and Psychiatry and Allied Disciplines, 33, 649-657.

Henderson, D. \& Vandenberg, B. (1992). Factors influencing adjustment in the families of autistic children. Psychological Reports, 71, 167-171.

Hindley, P. (1997). Psychiatric aspects of hearing impairments. Journal of Child Psychology and Psychiatry and Allied Disciplines, 38, 101-117.

Howlin, P. \& Clements, J. (1995). Is it possible to assess the impact of abuse on children with pervasive developmental disorders? Journal of Autism and Developmental Disorders, 25, 337-354.

Jones, G. (1997). Disabling children: autism, the effect on families and professionals. Educational and Child Psychology, 14, 71-76.

Kaiser, A. P., Hester, P. P. \& McDuffie, A. S. (2001). Supporting communication in young children with developmental disabilities. Mental Retardation and Developmental Disabilities Research Reviews, 7, 143-150.
Kanner, L. (1943). Autistic disturbances of affective contact. Nervous Child, 2, $217-$ 250.

Kanner, L. (1971). Follow-up study of eleven autistic children originally reported in 1943. Journal of Autism and Childhood Schizophrenic, 1, 119-145.

Kanner, L., Rodriguez, A. \& Ashenden, B. (1972). How far can autistic children go in matters of social adaptation? Journal of Autism and Childhood Schizophrenia, 2, 9-33.

Kasari, C. \& Sigman, M. (1997). Linking parental perceptions to interactions in young children with autism. Journal of Autism and Developmental Disorders, 27, $39-57$.

Klein, R. G. \& Slomkowski, C. (1993). Treatment of psychiatric disorders in children and adolescents. Psychopharmacological Bulletin, 29, 525-535.

Koegel, R. L., Bimbela, A. \& Schreibman, L. (1996). Collateral effects of parent training on family interactions. Journal of Autism and Developmental Disorders, 26, 347-359.

Koegel, R. L., Schreibman, L., Loos, L. M., Dirlich-Wilhelm, H., Dunlap, G., Robbins, F. R. \& Plienis, A. J. (1992). Consistent stress profiles in mothers of children with autism. Journal of Autism and Developmental Disorders, 22, 205-216.

Konstantareas, M. M. (1991). Autistic, learning disabled and delayed children's impact on their parents. Canadian Journal of Behavioural Science, 23, 358-375.

Konstantareas, M. M., Homatidis, S. \& Cesaroni, L. (1995). Brief report: Variables related to parental choice to medicate their autistic children. Journal of Autism and Developmental Disorders, 25, 443-452.

Konstantareas, M. M., Homatidis, S. \& Plowright, C.M. (1992). Assessing resources and stress in parents of severely dysfunctional children through the Clarke modification of Holroyd's Questionnaire on Resources and Stress. Journal of Autism and Developmental Disorders, 22, 217-234.

Kracke, I. (1994). Developmental prosopagnosia in Asperger syndrome: presentation and discussion of an individual case. Developmental Medicine Cbild Neurology, 36, 873-886.

Kuloglu Aksaz, N. (1994). The effect of informational counseling on the stress level of parents of children with autism in Turkey. Journal of Autism and Developmental Disorders, 24, 109-110.

Kupfer, M. C. M. (1997). Modelos de atendimento à criança psicótica. Boletim de Psicologia, 48, 77-96.

Lino Silva, M. E. (1998). Gente estranha: Um olhar psicanalítico para o autista. Estudos de Psicologia, 15, 59-67.

Lipp, M. E. N. (1989). O controle do stress. Campinas: Centro Psicológico de Controle do Stress. (Manuscrito não-publicado)

Lipp, M. E. N. \& Guevara, A. J. (1994). Validação empírica do Inventário de Sintomas de Stress (ISS). Estudos de Psicologia, 3/4, 6-19.

Lipp, M. E. N. \& Rocha, J. C. (1994). Stress, hipertensão arterial e qualidade de vida. Campinas, SP: Papirus.

Lord, C., Rutter, M. \& Le Couteur, A. (1994). Autism Diagnostic Interview-Revised: Revised version of a diagnostic interview for caregivers of individuals with possible pervasive developmental disorders. Journal of Autism and Developmental Disorders, 24, 659-685.

Mesibov, G., Schopler, E., Schaffer, B. \& Landrus, R. (1988). Adolescent and Adult Psychoeducational Profile (AAPE), (Vol. IV). Texas: Pro-Ed.

Mickelson, K. D., Wroble, M. \& Helgeson, V. S. (1999). 'Why my child?': Parental attributions for children's special needs. Journal of Applied Social Psychology, 29, 1263-1292.

Moes, D., Koegel, R. L., Schreibman, L. \& Loos, L. M. (1992). Stress profiles for mothers and fathers of children with autism. Psychological Reports, 71, 12721274.

Morales, E., Martinez, R. \& Váldes, A. (1995). Un modelo de acción con un miembro autista. Revista de Psiquiatria (Chile), 12, 26-33.

Nally, B., Houlton, B. \& Ralph, S. (2000). The management of television and video by parents of children with autism. Autism, 4, 331-337.

Organização Mundial da Saúde (1993). Classificação de transtornos mentais e de comportamento da CID-10: Descrições clínicas e diretriz̧es diagnósticas (D. Caetano, Trad.). Porto Alegre: ArtMed. 
Ornitz, E. M., Ritvo, E. R. \& Gauderer, E. C. (1987). Autismo: Revisão crítica da literatura. Em J. R. S. Lipp (Orgs.), Psiquiatria infantil: Estudo multidisciplinar (pp. 113-134). Belo Horizonte: ABENEDI.

Pereira, A. M. S. (2001). Resiliência, personalidade, stress e estratégias de coping. Em J. Tavares (Org.), Resiliência e educação (pp. 79-94). São Paulo: Cortez.

Perry, A. \& Black, A. (1997). A prospective study of out-of-home placement tendency in families of children with autism. Journal on Developmental Disabilities, 5,1-23.

Peterson, C. C. \& Siegal, M. (2000). Insights into theory of mind from deafness and autism. Mind and Language, 15, 123-145.

Piccinini, C. A., Moura, M. L. S., Ribas, A. F. P., Bosa, C. A., Oliveira, E. A., Pinto, E. B., Schermann, L. \& Chahon, V. L. (2001). Diferentes perspectivas na análise da interação pais-bebê/criança. Psicologia: Reflexão e Crítica, 14, 469-485.

Piven, J. \& Palmer, P. (1999). Psychiatric disorder and the broad autism phenotype: Evidence from a family study of multiple-incidence autism families. American Journal of Psychiatry, 56, 557-563.

Pomeroy, J. C. (1992). Autismo infantil e psicose da infância. Em B. D. Garfinkel, G. A. Carlson \& E. B. Weller (Orgs.), Transtornos psiquiátricos na infância e adolescência (pp. 235-250). Porto Alegre: ArtMed.

Powers, M. D. (1991). Intervening with families of young children with severe handicaps: Contributions of a family systems approach. School Psychology Quarterly, 6, 131-146.

Prado, M. C. C. (1999). Entrando em contato com o mundo da família autista: em busca de meios de comunicação. Informe Psiquiátrico 18, 3-13.

Reid, S. (1999). The assessment of the child with autism: A family perspective. Clinical Child Psychology and Psychiatry, 4, 63-78.

Rodrigue, J. R., Morgan, S. B. \& Geffken, G. R. (1992). Psychosocial adaptation of fathers of children with autism, Down syndrome, and normal development. Journal of Autism and Developmental Disorders, 22, 249-263.

Rossetti Ferreira, M. C. (1984). O apego e as reações da criança à separação da mãe: Uma revisão bibliográfica. Caderno de Pesquisa, 48, 3-19.

Sanders, J. L. \& Morgan, S. B. (1997). Family stress and adjustment as perceived by parents of children with autism or Down Syndrome: Implications for intervention. Child and Family Behavior Therapy, 19, 15-32.
Savóia, M. G. (1999). Escalas de eventos vitais e de estratégias de enfrentamento (coping). Revista de Psiquiatria Clinica, 26, 57-67.

Selye, H. (1956). The stress of life. New York: McGraw Hill.

Shu, B. C, Lung, F. W \& Chan, Y. Y. (2000). The mental health in mothers with autistic children: A case-control study in southern Taiwan. Kaobsiung Journal Medicine Science, 16, 308-314.

Sidoli, M. (2000). The little puppet: Working with autistic defences in motherinfant psychotherapy. Journal of Analytical Psychology, 45, 159-175.

Sprovieri, M. H. S. \& Assumpção Jr, F. B. (2001). Dinâmica familiar de crianças autistas. Arquivo Neuropsiquiátrico, 79, 230-237.

Szatmari, P., Archer, L., Fisman, S. \& Streiner, D. L. (1994). Parent and teacher agreement in the assessment of pervasive developmental disorders. Journal of Autism and Developmental Disorders, 24, 703-717.

Travis, L. L. \& Sigman, M. (1998). Social deficits and interpersonal relationships in autism. Mental Retardation and Developmental Disabilities Research Reviews, 4, 6572.

Triviños, A. N. S. (1987). Introdução à pesquisa em ciências sociais: A pesquisa qualitativa em educação. São Paulo: Atlas.

Tunali, B. \& Power, T. G. (1993). Creating satisfaction: a psychological perspective on stress and coping in families of handicapped children. Journal of Child Psychology and Psychiatric and Allied Disciplines, 34, 945-957.

Whiteley, P., Rodgers, J. \& Shattock, P. (1998). Clinical features associated with autism: observations of symptoms outside the diagnostic boundaries of autistic spectrum disorders. Clinical features and autism, 2, 415-422.

Willemsen-Swinkels, S. H., Buitelaar J. K. \& van Engeland, H. (1997). Children with a pervasive developmental disorder, children with a language disorder and normally developing children in situations with high- and low-level involvement of the caregiver. Journal of Child Psychology and Psychiatric and Allied Disciplines, 38, 327-336.

Sobre os autores

Recebido: 08/08/2003

Última revisão: 26/05/2004

Aceite final: 20/08/2004

Maria Ângela Bravo Fávero é Psicóloga, graduada pela Faculdade de Filosofia, Ciências e Letras de Ribeirão Preto (FFCLRP) da Universidade de São Paulo, mestre do Programa de Pós-Graduação em Psicologia do Departamento de Psicologia e Educação da FFCLRP-USP, pesquisadora da Fundação de Amparo à Pesquisa do Estado de São Paulo (FAPESP).

Manoel Antônio dos Santos é Psicólogo, Doutor em Psicologia Clínica pela Universidade de São Paulo. : Doutor em Psicologia Clínica pela Universidade de São Paulo, Professor Doutor do Programa de Pós-Graduação em Psicologia do Departamento de Psicologia e Educação da FFCLRP-USP, pesquisador do CNPq. 